\title{
Gas Filled RF Resonator Hadron Beam Monitor for Intense Neutrino Beam Experiments
}

\author{
K. Yonehara* \\ Fermilab, \\ E-mail: yoneharaefnal.gov
}

\section{M.A. Cummings ${ }^{\dagger}$}

Muons, Inc.

E-mail: macc@muonsinc.com

\author{
R.P. Johnson \\ Muons, Inc. \\ E-mail: rol@muonsinc.com
}

\begin{abstract}
The Long Baseline Neutrino Facility (LBNF) is the future flagship project at Fermilab to reveal nature of neutrino. Beam profile monitors (BPM) in the front-end of neutrino beam line play an important role to measure quality of the secondary charged particles and to precisely direct the beam to the neutrino detector located hundreds of kilometers away through a secondary-beam production target. One of the challenges to realize a multi-MW beam complex is monitoring of the beam profile under extreme radiation environments. We propose a radiation-tolerant gas-filled multi-RF cavity beam profile monitor that provides precise measurements of the beam downstream of a secondary-particle production target. The RF monitor is based on microwave cavity resonators that are a simple metallic box filled with gas. Charged particles passing through each RF cavity in the monitor produce intensity-dependent ionized plasma which changes the gas permittivity. The permittivity change is measured by observing the RF quality factor change and the resonant frequency shift in the resonator. The beam profile is reconstructed from the RF signal in individual RF cavity of the monitor. Analytical and numerical studies have been made. A part of numerical results is shown in the document.
\end{abstract}

38th International Conference on High Energy Physics

3-10 August 2016

Chicago, USA

${ }^{*}$ Work supported by Fermilab Research Alliance, LLC under Contract No. DE-AC02-07CH11359 and DOE STTR Grant, No. DE-SC0013795.

${ }^{\dagger}$ Speaker. 


\section{Introduction}

Figure 1 shows the current NuMI front end which has a typical beam component to produce intense neutrino fluxes. There are two kinds of BPMs to diagnose the quality of secondary and tertiary particle fluxes, called hadron and muon monitors. A hadron monitor is located at the end of a decay pipe to directly measure the profile of secondary particles while a muon monitor is located downstream of the absorber which eliminates most particles except for muons. The hadron monitor is heavily exposed to the beam radiation. Indeed, the present hadron monitor which is based on an ionization chamber loses signal gain by radiation damage and its lifetime is $3 \sim 4$ years in the NuMI application. In case of LBNF [1], the design beam power is $2.4 \mathrm{MW}(0.5-0.7 \mathrm{MW}$ for NuMI). A hadron monitor for LBNF will be located $200 \mathrm{~m}$ downstream of the target. A new type of radiation hard hadron monitor is required.

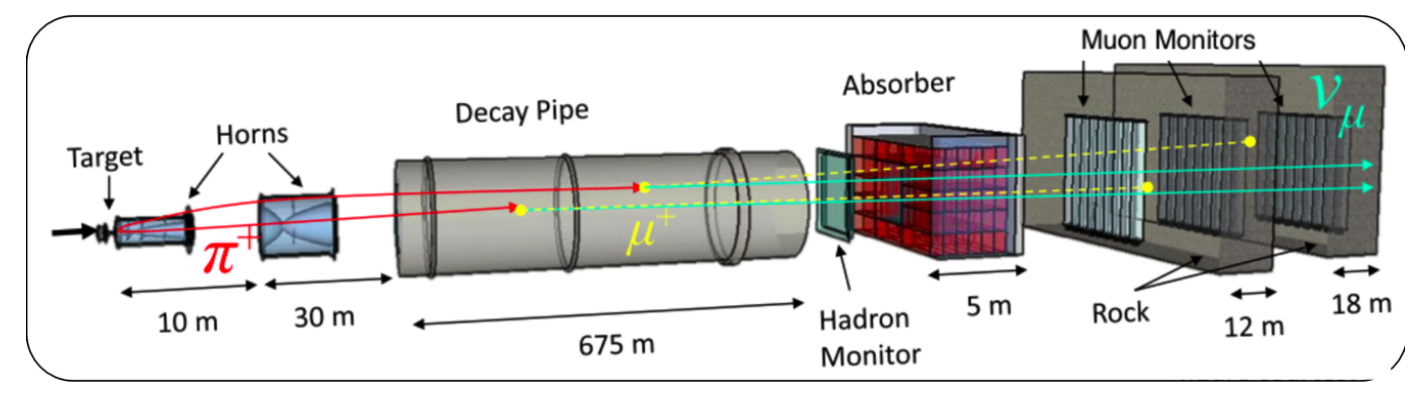

Figure 1: Layout of the current NuMI front end. There are three horns and a short decay pipe for LBNF.

A novel multi-RF cavity gas monitor is proposed. A beam-induced plasma changes the gas permittivity. A size of gas permittivity is also changed by a RF amplitude and gas pressure. The change is read-out by the RF signal that is modulated by the quality factor and resonant frequency shifts. Only a thin-metal chamber with gas is exposed to the beam radiation and other devices like the RF read-out system are outside the beam enclosure. The concept has been tested in analytical and numerical studies [2]. The sensitivity of the RF gas monitor as functions of the target density change and various sizes of primary beam is demonstrated in the document.

\section{Numerical Simulation Study}

\subsection{Condition in simulation}

The RF quality factor change is evaluated in this demonstration. G4Beamline is used for an event generator [3]. A 1-meter-long carbon target is used in simulation. The transverse beam emittance is $30 \pi \mathrm{mm}$-mrad. For simplicity, no horn nor a focusing field is implemented and the RF monitor is evaluated only by protons in simulation since the yield of secondary charged particles is dominated by high energy protons. A phase space of protons is collected and the probability densify function is generated at the place of hadron monitor. The RF monitor is modeled as a $12 \times 12$-pixel gas-filled RF cavity. Geometry of each cavity is $30 \times 30 \times 10 \mathrm{~mm}^{3}$. The cavity will be re-entrant shape to excite with $2.45 \mathrm{GHz}$, which is a standard frequency of a RF source.

The gas permittivity change is given by the population of plasma and its dynamics in RF fields. It is controlled by a gas pressure, RF gradient, and concentration of electronegative dopant in the 
cavity. Optimization of the RF parameter is done based on the past RF measurements [4,5]. Two operation modes are considered to diagnose the beam quality. The beam intensity will be extremely low for a beam commissioning. Horns and target positions are checked by scanning the position of low intensity beam. A pure atmospheric nitrogen gas will be used to increase the sensitivity of the RF gas monitor. The RF amplitude will be $0.1 \mathrm{MV} / \mathrm{m} .10^{6}$ charged particles per pulse in $\mathrm{cm}^{2}$ can be measured in the RF gas monitor. The required RF power per pixel is a few Watts which is marginal for a standard RF source.

In case of the nominal beam operation, the plasma density in the cavity will reach to $\sim 10^{11}$ $\mathrm{cm}^{3}$. The RF quality factor becomes significantly small because the RF power dissipation in ionized electrons becomes significant. To avoid such a power loss, a small amount of electronegative dopant is added in the cavity. The gas pressure increment will be required to enhance the electron capture rate. Unfortunately, the electron capture rate in the range of RF gas monitor at the normal beam operation has not measured in past. The demonstration test is planed and the first test will be carried out in 2017.

\subsection{Simulation results}

Only the low beam intensity mode is carried out in the demonstration. Figure 2 shows the estimated RF signal in the RF gas monitor. From simulation study, the estimated beam centroid

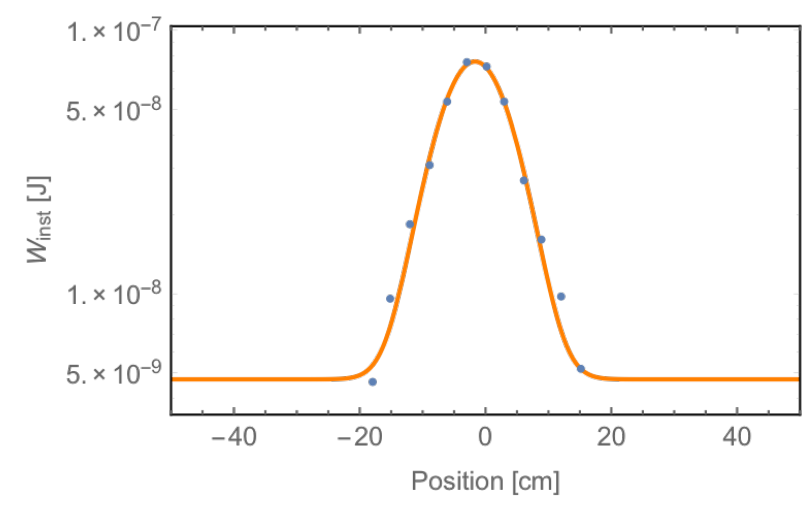

Figure 2: Estimated RF signal in the RF gas monitor. The RMS of the RF signal is $7.2 \mathrm{~cm}$.

position sensitivity in the RF gas monitor is $0.5 \mathrm{~mm}$. If the target center is a pivot point of the beam, the sensitivity of beam tilting angle is $2.5 \mu \mathrm{rad}$.

The RF gas monitor is possibly sensitive to the target density and the size of primary proton beam on target. Figure 3 shows the estimated RF signal in the RF gas monitor as functions of target density and the size of primary proton beam. The RF noise in the RF detector system should be involved in this analysis. From past RF measurements, the absolute beam intensity measurement has $10 \%$ errors. If the measurement error is similar as the past measurement, $4 \%$ of target density reduction will be detectable. The error study will be done in the demonstration test.

\section{Conclusion}

Evaluation of the RF gas monitor has been done in numerical simulation. The result showed that the concept of RF gas monitor has a capability to apply for the LBNF front end. As the 

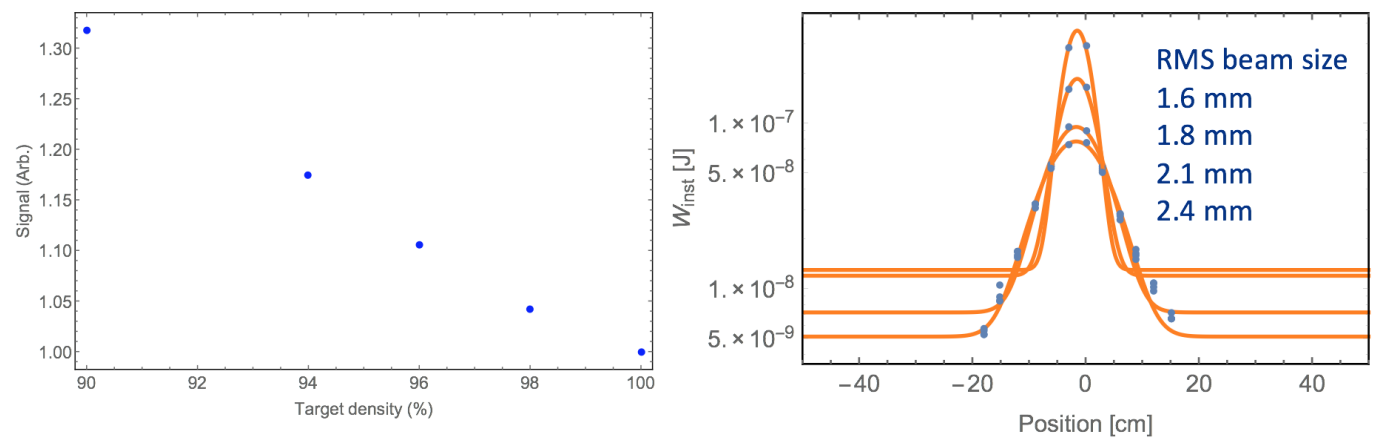

Figure 3: The left-hand-side plot shows the estimated RF signal in the RF gas monitor as a function of target density. The right-hand-side plot shows the estimated RF signal in the RF gas monitor as a function of primary beam size.

next step, feasibility of the monitor should be made. It is crucial to measure the electron capture time to estimate the sensitivity of RF gas monitor as a function of beam intensity. Shorter electron capture time is better to reduce the measurement error in the RF gas monitor. Past RF measurement suggests that the electron capture time can be shorter than $1 \mathrm{~ns}$ with a $1 \% \mathrm{O}_{2}$ doped 20-atm nitrogen gas. Higher RF power is needed to recover an observable RF signal size in the cavity if the capture time exceeds $1 \mathrm{~ns}$. Fortunately, the capacity of RF source is marginal to compensate the RF power dissipation in plasma.

A RF waveguide, power divider, LLRF controller and RF signal analyzer should be involved to design a whole RF system. In the original design, single RF source will provide whole RF power into the monitor. However, the RF power loading in each cavity should be different. We should consider how to balance the RF power and how to send the RF power into individual RF cavity through the divider.

Temperature on the monitor is increased due to the beam energy deposition. Fortunately, the increment is a few degree for the nominal beam operation [6]. However, for the false condition, temperature can grow 100 degrees or higher because the primary beam can directly hit the monitor. The cavity geometry change due to thermal expansion should be involved in the design.

\section{References}

[1] "The Long-Baseline Neutrino Facility for DUNE", CDR Volume 3,

[2] K. Yonehara et al., "Progress of Gas-Filled Multi-RF-Cavity Beam Profile Monitor for Intense Neutrino Beams", Proc. NAPAC16, to be published.

[3] G4Beamline, http://www.muonsinternal.com/muons3/G4beamline

[4] M. Chung et al., "Pressurized H2 rf Cavities in Ionizing Beams and Magnetic Fields", Phy. Rev. Lett. $111,184802,2013$.

[5] B. Freemire et al., "Pressurized rf Cavities in Ionizing Beams", Phy. Rev. Acc. Beams 19, 062004, 2016.

[6] Q. Liu et al., "Progress of Gas-Filled Multi-RF-Cavity Beam Profile Monitor for Intense Neutrino Beams", Proc. NAPAC16, to be published. 